\title{
A STUDY OF MAGNETIC BRIGHT POINTS IN THE Na I D 1 LINE
}

\author{
D. B. Jess ${ }^{1}$, M. Mathioudakis ${ }^{1}$, D. J. Christian ${ }^{2}$, P. J. Crockett ${ }^{1}$, and F. P. Keenan ${ }^{1}$ \\ ${ }^{1}$ Astrophysics Research Centre, School of Mathematics and Physics, Queen's University, Belfast, BT7 1NN, UK; d.jess@ qub.ac.uk \\ ${ }^{2}$ Department of Physics and Astronomy, California State University, Northridge, CA 91330, USA \\ Received 2010 June 7; accepted 2010 July 9; published 2010 July 28
}

\begin{abstract}
High-cadence, multiwavelength, optical observations of solar magnetic bright points (MBPs), captured at the disk center using the ROSA and IBIS imaging systems on the Dunn Solar Telescope, are presented. MBPs manifesting in the $\mathrm{NaI} \mathrm{D}_{1}$ core are found to preferentially exist in regions containing strong downflows, in addition to cospatial underlying photospheric magnetic field concentrations. Downdrafts within $\mathrm{Na}_{\mathrm{I}} \mathrm{D}_{1}$ bright points exhibit speeds of up to $7 \mathrm{~km} \mathrm{~s}^{-1}$, with preferred structural symmetry in intensity, magnetic field, and velocity profiles about the bright point center. Excess intensities associated with $G$-band and Ca II K observations of MBPs reveal a power-law trend when plotted as a function of the magnetic flux density. However, Na I $\mathrm{D}_{1}$ observations of the same magnetic features indicate an intensity plateau at weak magnetic field strengths below $\approx 150 \mathrm{G}$, suggesting the presence of a two-component heating process: one which is primarily acoustic and the other predominantly magnetic. We suggest that this finding is related to the physical expansion of magnetic flux tubes, with weak field strengths $(\approx 50 \mathrm{G})$ expanding by $\sim 76 \%$, compared to a $\sim 44 \%$ expansion when higher field strengths $(\approx 150 \mathrm{G})$ are present. These observations provide the first experimental evidence of rapid downdrafts in $\mathrm{Na}$ I $\mathrm{D}_{1}$ MBPs and reveal the nature of a previously unresolved intensity plateau associated with these structures.
\end{abstract}

Key words: Sun: atmosphere - Sun: chromosphere - Sun: photosphere - Sun: surface magnetism

\section{INTRODUCTION}

In solar physics, the $\mathrm{Na}_{\mathrm{I}} \mathrm{D}_{1}$ absorption profile has long been a difficult line to study and indeed model. Line profile fluctuations often result from a combination of Doppler wavelength shifts, $p$-mode oscillations, and other small-scale dynamical phenomena (Edmonds \& Hsu 1983). Furthermore, temperature, microturbulence, and magnetic field strength can affect the associated Na I $\mathrm{D}_{1}$ line depths (Athay \& Canfield 1969; Slaughter \& Wilson 1972). The formation height of the $\mathrm{Na}$ I $\mathrm{D}_{1}$ core has often been the most difficult to interpret. Aimanova \& Gulyaev (1976) used slitless spectrograms to estimate a core formation height of $1300-1700 \mathrm{~km}$. This is above the formation height of the Ca II K core $(\approx 1200 \mathrm{~km}$; Beebe \& Johnson 1969) and overlaps with that of the $\mathrm{H} \alpha$ core $(\approx 1500 \mathrm{~km}$; Vernazza et al. 1981). However, observations indicate that $\mathrm{Na}_{\mathrm{I}} \mathrm{D}_{1}$ core images have little in common with their $\mathrm{Ca}$ II $\mathrm{K}$ or $\mathrm{H} \alpha$ counterparts. Eibe et al. (2001) have utilized theoretical $\mathrm{Na}$ I $\mathrm{D}_{1}$ response functions to suggest that the formation height varies depending on the local temperature and/or velocity. The authors estimate a Na I $\mathrm{D}_{1}$ core formation height of $<1000 \mathrm{~km}$, based upon the non-local thermodynamic equilibrium radiative transfer code of Carlsson (1986). This is consistent with the work of Tomita (1960), Finsterle et al. (2004), and Simoniello et al. (2008), who suggest $\mathrm{Na}$ I $\mathrm{D}_{1}$ line-core emission originates from the upper photospheric layer $(<800 \mathrm{~km})$.

Recent $\mathrm{Na}_{\mathrm{I}} \mathrm{D}_{1}$ observations and simulations have revealed that $\mathrm{Na}$ I $\mathrm{D}_{1}$ core brightenings sample magnetic field concentrations in the quiet solar network (Leenaarts et al. 2010). Through a comparison of simultaneous MDI magnetograms with groundbased $\mathrm{Na}_{\mathrm{I}} \mathrm{D}_{2}$ observations, Cauzzi et al. (2000) were able to suggest that sodium bright points are coincident in position, size, and shape with corresponding magnetic patches. However, the relatively poor spatial resolution of MDI $\left(\approx 1^{\prime \prime} .2\right)$ limited the study to network bright points in excess of $\approx 3^{\prime \prime}$ photospheric diameter. Thus, it is imperative to study these types of bright features with an imaging system capable of high spatial resolution. In this Letter, we utilize a high-cadence multiwavelength data set to search for correlations linking small-scale $\mathrm{Na} \mathrm{D}_{1}$ intensity enhancements to the underlying photospheric magnetic field.

\section{OBSERVATIONS}

The data presented here are part of an observing sequence obtained during 13:46-14:47 UT on 2009 May 28, with the Dunn Solar Telescope at Sacramento Peak, New Mexico. The Rapid Oscillations in the Solar Atmosphere (ROSA; Jess et al. 2010b) six-camera system was employed in conjunction with the Interferometric BIdimensional Spectrometer (IBIS; Cavallini 2006) to image a $69.3 \times 69$ '. 1 region positioned at the solar disk center. IBIS sampled the $\mathrm{NaI} \mathrm{D}_{1}$ absorption line at $5895.94 \AA$, incorporating nine wavelength steps with 10 exposures per step to assist image reconstruction. More details of our instrumentation setup are given in Table 1. A spatial sampling of 0.069 pixel $^{-1}$ was used for the ROSA cameras to match the telescope's diffraction-limited resolution in the blue continuum to that of the CCD. This results in images obtained at longer wavelengths being slightly oversampled. However, this was deemed desirable to keep the dimensions of the field of view the same for all ROSA cameras. IBIS employed a spatial sampling of 0 '.083 pixel $^{-1}$, which allowed ROSA's near square field-of-view to be contained within the circular aperture provided by IBIS.

During the observations, high-order adaptive optics (Rimmele 2004) were used to correct wavefront deformations in real time. The acquired images were further improved through speckle reconstruction algorithms (Weigelt \& Wirnitzer 1983; Wöger et al. 2008), utilizing $16 \rightarrow 1$ and $10 \rightarrow 1$ restorations for ROSA and IBIS images, respectively. Post-reconstruction cadences for ROSA and IBIS are displayed in the fourth column of Table 1. A full image-reconstructed IBIS scan through the $\mathrm{Na} \mathrm{I}_{1}$ absorption line resulted in a cadence of $39.703 \mathrm{~s}$ and includes a blueshift correction required due to the use of classical 
Table 1

ROSA/IBIS Filter and Cadence Overview

\begin{tabular}{lccc}
\hline \hline \multicolumn{1}{c}{ Filter Used } & $\begin{array}{c}\text { Exposure Time } \\
(\mathrm{ms})\end{array}$ & $\begin{array}{c}\text { Frames per } \\
\text { Second }\end{array}$ & $\begin{array}{c}\text { Reconstructed } \\
\text { Cadence (s) }\end{array}$ \\
\hline$G$ band & 15 & 30.3 & 0.528 \\
Blue continuum $(4170 \AA)$ & 10 & 30.3 & 0.528 \\
Ca II K core & 200 & 3.8 & 4.224 \\
$\mathrm{H} \alpha$ core (Zeiss) & 240 & 3.8 & 4.224 \\
$\mathrm{LCP}(6302.375 \AA)$ & 240 & 3.8 & 4.224 \\
$\mathrm{RCP}^{\mathrm{b}}(6302.375 \AA)$ & 240 & 3.8 & 4.224 \\
$\mathrm{Na}$ I D $\mathrm{D}_{1}(5895.94 \AA)$ & 70 & $2.3^{\mathrm{c}}$ & $39.703^{\mathrm{d}}$ \\
\hline
\end{tabular}

Notes.

${ }^{a}$ Left-hand circularly polarized light.

${ }^{\mathrm{b}}$ Right-hand circularly polarized light.

${ }^{c}$ Average frames per second including Fabry-Perot tuning time.

d Cadence of a complete Na I $\mathrm{D}_{1}$ profile scan.

etalon mountings (Cauzzi et al. 2008). To insure accurate coalignment in all bandpasses, broadband time series were Fourier co-registered and de-stretched using a $40 \times 40$ grid, equating to $\mathrm{a} \approx 1^{\prime \prime} .7$ separation between spatial samples (Jess et al. 2007, 2008). Narrowband images, including those from IBIS, were corrected by applying destretching vectors established from simultaneous broadband reference images (Reardon \& Cavallini 2008). Line-of-sight magnetograms were generated as a difference image, normalized to their sum, of left- and right-hand circularly polarized light, obtained $125 \mathrm{~m} \AA$ into the blue wing of the magnetically sensitive Fe I absorption line at $6302.5 \AA$. Utilizing high-resolution MDI magnetograms of a small active region, obtained in conjunction with ROSA using an identical optical setup, and following the previous work of Ishikawa et al. (2007), these normalized difference images were subsequently scaled to provide calibrated line-of-sight magnetograms. Due to the ROSA cameras remaining within their linear regime, and MDI observations residing below their saturation threshold, only linear corrections were required to produce calibrated line-of-sight magnetograms (D. B. Jess et al. 2010 , in preparation).

\section{ANALYSIS AND DISCUSSION}

No active regions were present on disk during our observations, and the ROSA/IBIS field-of-view contained no largescale magnetic activity. However, a small collection of magnetic bright points (MBPs) were visible at heliocentric coordinates $\left(3.9,-1^{\prime \prime} .5\right)$, providing an opportunity to examine these kiloGauss structures without the line-of-sight effects associated with near-limb observations. Figure 1 shows snapshots of all the ROSA/IBIS sub-images obtained at 14:06:21 UT, and reveals a collection of intensity enhancements, visible in the core of the $\mathrm{Na}$ I $\mathrm{D}_{1}$ line (lower-middle panel of Figure 1). Ca II K observations were acquired through a $1 \AA$ wide filter, with resulting emission originating from the upper photosphere to the lower chromosphere (see, e.g., the lower-right panel of Figure 1). The $\mathrm{Na}$ I $\mathrm{D}_{1}$ core image is an intensity map, created by establishing the line-profile minimum per pixel. By displaying Dopplercompensated line-center intensities, rather than rest-wavelength line-center intensities, brightness variations throughout the image are more indicative of the source function than of the velocities present in the line-forming region (Leenaarts et al. 2010). Examination of $G$-band (upper-left panel of Figure 1) and Na I $\mathrm{D}_{1}$ core images reveal, crucially, that not all $\mathrm{Na}$ I $\mathrm{D}_{1}$ core brightenings can be directly linked to underlying MBPs. Thus, there may be two possible interpretations which promote efficient $\mathrm{Na}$ I $\mathrm{D}_{1}$ core brightening. The first implies independent heating processes, whereby magnetic and non-magnetic brightenings are produced via unrelated mechanisms. Contrarily, another interpretation revolves around the same heating mechanism, but that the process is somehow more effective in highly magnetic areas, such as those found in MBP locations. Therefore, it is important to investigate small-scale MBPs and establish which process allows enhancement of the $\mathrm{NaI} \mathrm{D}_{1}$ line above these $<2^{\prime \prime} G$-band structures.

Information contained within the $\mathrm{Na} \mathrm{I}_{1}$ core velocity maps, generated through Doppler shifts of the profile minimum, begins to unlock this mystery. An inspection of Figure 1 reveals a preference for NaI $\mathrm{D}_{1}$ and $G$-band MBP structures to exist in downflow regions (up to $7 \mathrm{~km} \mathrm{~s}^{-1}$ ). Furthermore, Ca II K core and $\mathrm{H} \alpha$ core intensity enhancements are often observed to coexist in locations of strong downflows $\left(>3 \mathrm{~km} \mathrm{~s}^{-1}\right)$ and longitudinal magnetic fields. Figure 2 shows a slice through a typical high redshift feature, displaying its $G$-band intensity, $\mathrm{Na}$ I $\mathrm{D}_{1}$ core intensity, magnetic field strength, and $\mathrm{Na}$ I $\mathrm{D}_{1}$ linecore velocity as a function of distance across the underlying MBP.

In the top-right panel of Figure 3, we present an averaged $\mathrm{Na}$ I $\mathrm{D}_{1}$ line profile (solid line) from the central region of an MBP, labeled "A" in Figure 1, where high redshifts are observed. A dashed line represents a $\mathrm{Na}$ I $\mathrm{D}_{1}$ profile, which has been spatially, and temporally, averaged over the entire field-of-view and time series (in excess of $9 \times 10^{7}$ individual profiles). It is evident that the MBP profile has been significantly Doppler shifted when compared to the average $\mathrm{NaI} \mathrm{D}_{1}$ profile. The difference between these two profiles is displayed in the bottomright panel of Figure 3 and reveals a blue bump at $\approx-4 \mathrm{~km} \mathrm{~s}^{-1}$, alongside a redshifted dip at $\approx 8 \mathrm{~km} \mathrm{~s}^{-1}$. These asymmetries are indicative of shock formation below the chromospheric canopy and are in remarkable agreement with the simulations of Leenaarts et al. (2010).

Roudier et al. (2006) investigated velocity flows associated with the $\mathrm{Na}$ I $\mathrm{D}_{1}$ line in strong magnetic field regions surrounding active regions and sunspots. These authors detected an abundance of downflows in these locations, with velocities nearing $2 \mathrm{~km} \mathrm{~s}^{-1}$. As shown in the lower-right panel of Figure 2, the $\mathrm{Na}$ I $\mathrm{D}_{1}$ core velocities presented here are considerably higher, reaching $\approx 7 \mathrm{~km} \mathrm{~s}^{-1}$ at the center of the MBP. Even artificially binning our data to mimic the resolution of Roudier et al. (2006) would not reduce the peak $\mathrm{Na}_{\mathrm{I}} \mathrm{D}_{1}$ Doppler velocities to below $3 \mathrm{~km} \mathrm{~s}^{-1}$. However, the spectral resolution of IBIS $(23 \mathrm{~m} \AA$ FWHM) is considerably better than the resolution obtained by the Multichannel Subtractive Double Pass system (144 mA FWHM) employed by Roudier et al. (2006) and allows a higher degree of precision when determining line-core velocities. Quantitatively, the Doppler velocity error, err, can be defined as err $= \pm \frac{\mathrm{FWHM} c}{2 \lambda}$ (Langangen et al. 2008), where $\lambda$ is the line-core wavelength and $c$ is the speed of light. This produces an associated IBIS velocity error of $\pm 0.5 \mathrm{~km} \mathrm{~s}^{-1}$, compared to an error of $\pm 3.7 \mathrm{~km} \mathrm{~s}^{-1}$ in the results of Roudier et al. (2006). In addition, $\mathrm{Na}$ I $\mathrm{D}_{1}$ downdraft velocities approaching $7 \mathrm{~km} \mathrm{~s}^{-1}$ are consistent with the magnetohydrodynamic simulations of Leenaarts et al. (2010). Thus, for the first time, the combined high spatial and spectral resolutions of speckle-reconstructed $\mathrm{Na}_{\text {I }} \mathrm{D}_{1}$ IBIS data sets allow fine-scale Doppler velocities to be evaluated with an unprecedented degree of precision.

From the lower panels of Figure 2, we see that MBP structures found in $\mathrm{Na}$ I $\mathrm{D}_{1}$ core imaging demonstrate an increased 

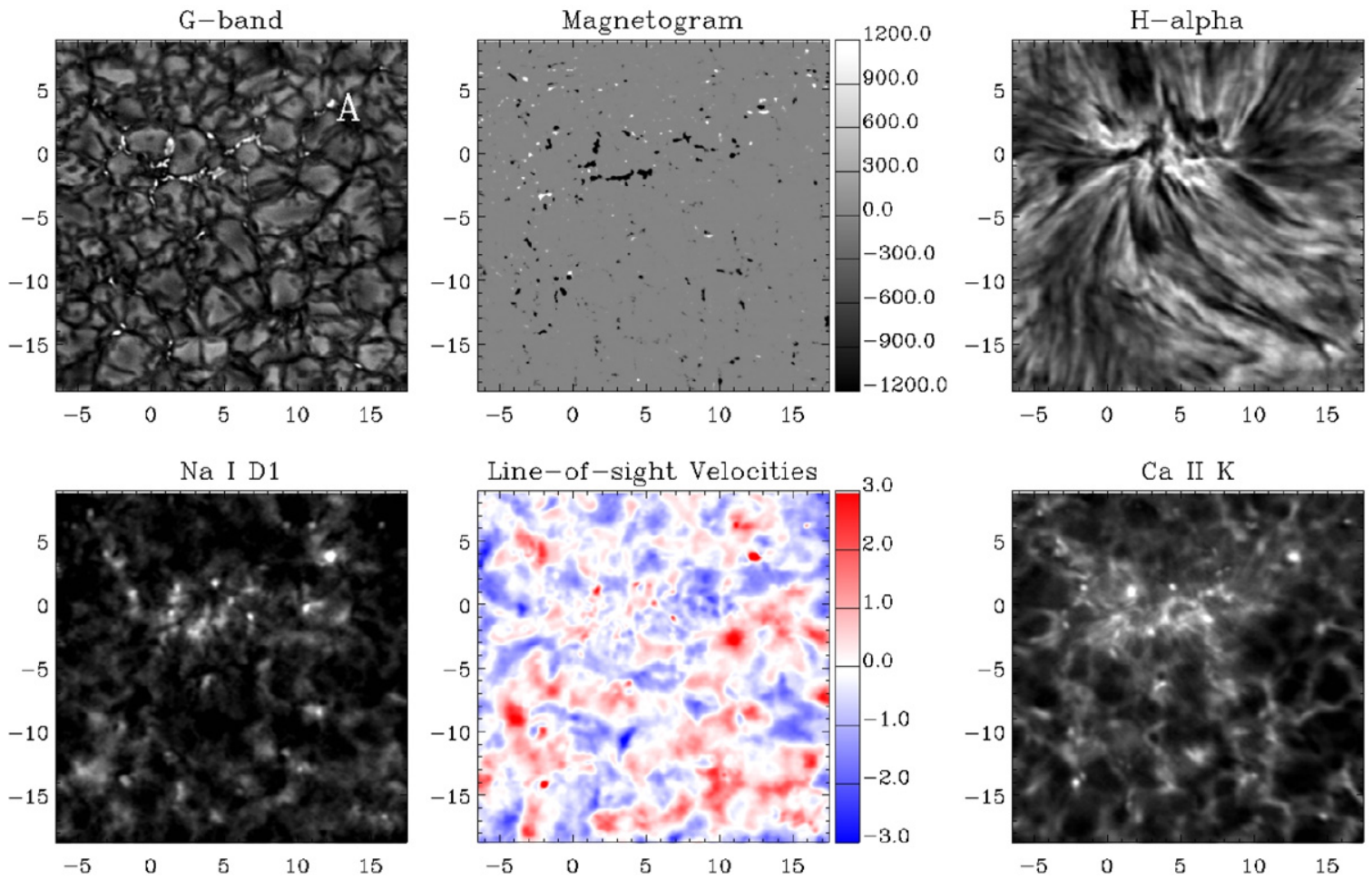

Figure 1. Images from ROSA/IBIS obtained simultaneously at 14:06:21 UT. Color scales for the line-of-sight magnetogram and velocity maps indicate magnetic field strength (in Gauss) and $\mathrm{Na}$ I $\mathrm{D}_{1}$ core velocity (in $\mathrm{km} \mathrm{s}^{-1}$ ), respectively. Artificial downdraft saturation is displayed in the velocity map to aid identification of MBP features. Axes are solar heliocentric coordinates in arcseconds. Properties related to the MBP labeled "A" are subsequently displayed in Figures 2 and 3.
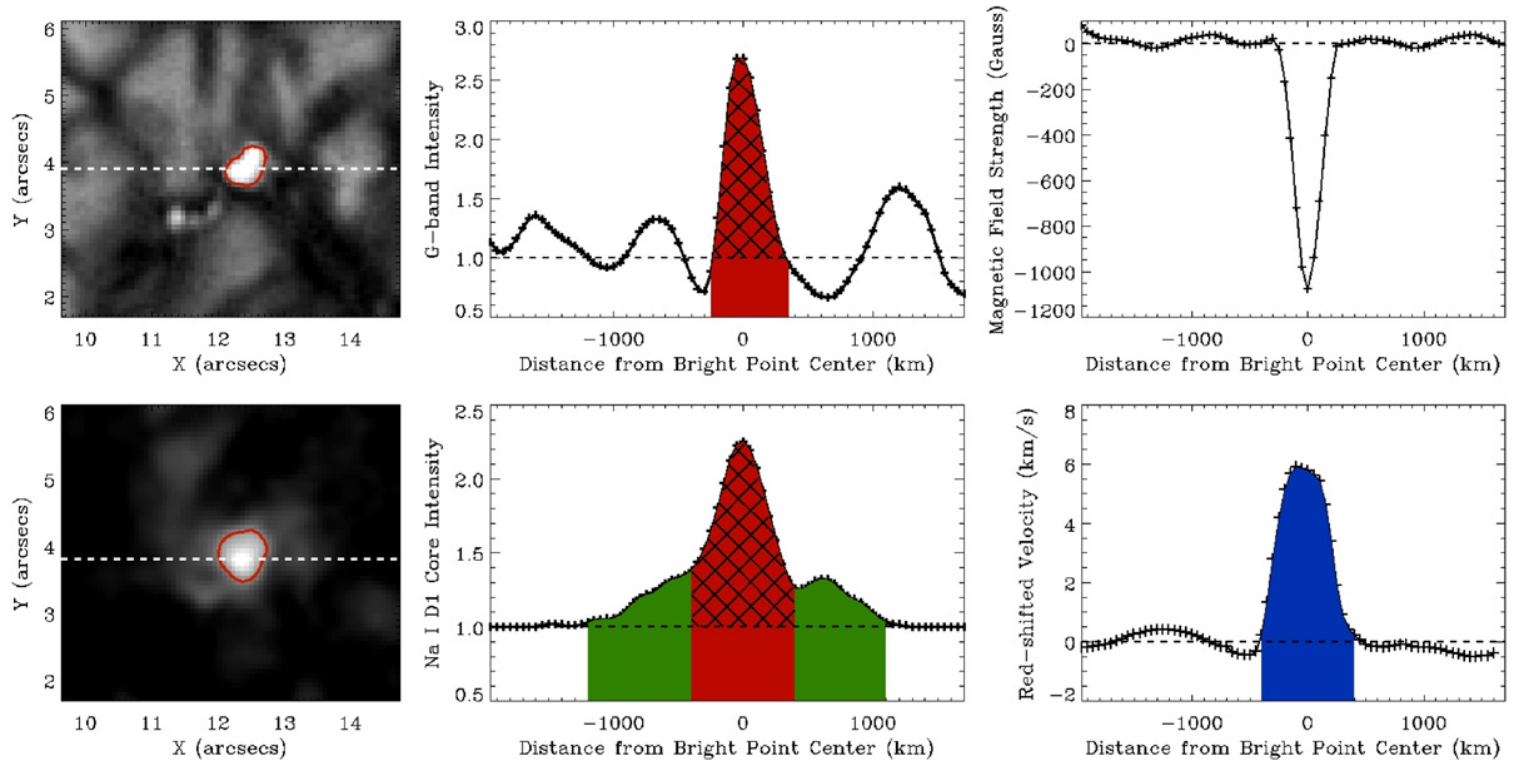

Figure 2. Left-hand panels show the $G$-band (upper) and Na I $\mathrm{D}_{1}$ core (lower) intensity images of the MBP labeled "A" in Figure 1. Overplotted in red are the pixels used to determine the excess intensity plotted in Figure 4. The remaining panels plot the one-dimensional variations in normalized $G$-band intensity (upper middle), normalized Na I $\mathrm{D}_{1}$ core intensity (lower middle), magnetic field strength (upper right), and $\mathrm{Na}$ I $\mathrm{D}_{1}$ core downdraft velocities (lower right), plotted as a function of distance from the center of the MBP along the cut indicated by the horizontal dashed lines in the left-hand panels. Green shading in the normalized Na I $\mathrm{D}_{1}$ core intensity plot indicates the extent of the halo around the MBP, while red shading marks the locations of the central MBP. Blue shading reveals regions of non-zero downdraft velocities, synonymous with $\mathrm{Na}$ I $\mathrm{D}_{1}$ core MBP enhancements. Cross-hatched regions display the values used when determining the excess intensity.

width over simultaneous $G$-band observations. This is consistent with the "aureoles," or halos, found by Leenaarts et al. (2010), who interpret these additional brightenings as an artifact of strong radiative scattering. It must be noted that the decrease in normalized intensity to values below 1.0, at the edges of the $G$-band MBP, is due to intensity sampling of the dark inter-granular lane, where the MBP resides. Exam- ination of Figure 2 suggests that the fastest downdrafts are cospatial with the center of the MBP and that the velocities drop to approximately zero at the edge of the structure. Furthermore, the strongest longitudinal magnetic field strength is also cospatial with the center of the MBP and correlates well in size with the width of the $G$-band structure. Following analysis of all MBPs observed with redshifts exceeding 

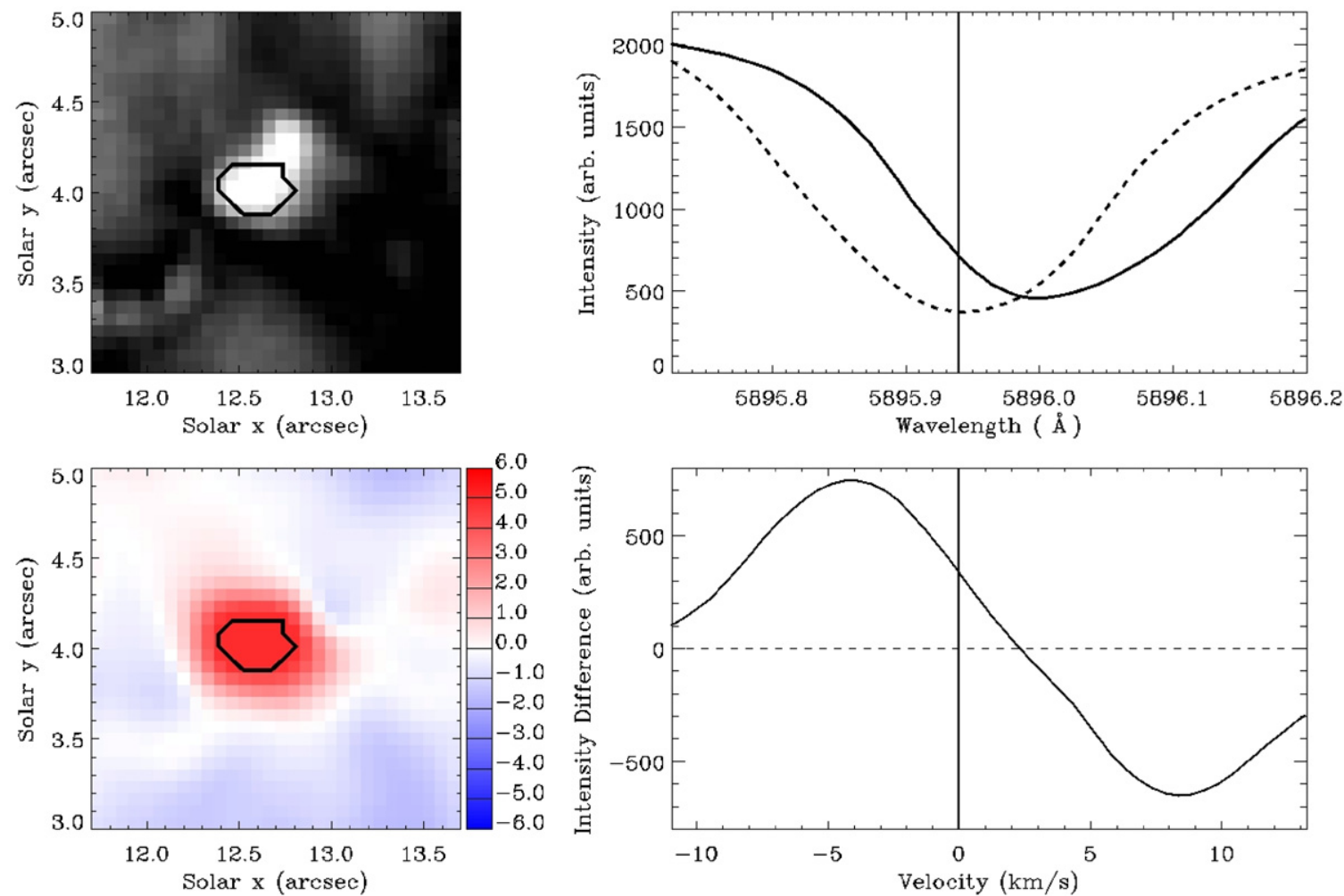

Figure 3. Top left: $G$-band intensity image of the MBP labeled "A" in Figure 1. Lower left: line-of-sight velocity map of the same MBP, obtained from Doppler wavelength shifts of the $\mathrm{Na}_{\mathrm{I}} \mathrm{D}_{1}$ profile. The color scale indicates flow velocities in $\mathrm{km} \mathrm{s}^{-1}$, where red and blue colors represent downflows and upflows, respectively. In each panel, the solid black contour indicates regions where downflow velocities exceed $4 \mathrm{~km} \mathrm{~s}^{-1}$. Top right: Averaged Na I $\mathrm{D}_{1}$ profile (solid line) generated from locations contained within the contours displayed in the left panels of this figure, overplotted with a rest-wavelength Na I $\mathrm{D}_{1}$ profile (dashed line). Lower right: the difference between the two profiles. In both right-hand panels, the solid vertical line represents the $\mathrm{Na}_{\mathrm{I}} \mathrm{D}_{1}$ rest wavelength at $5895.94 \AA$.

$3 \mathrm{~km} \mathrm{~s}^{-1}$ and underlying magnetic field concentrations, prevailing symmetry is found in intensity, velocity, and magnetic-field strength as a function of radial distance from the center of the MBP. This indicates the preference for an ordered structure, whereby maximum values are attributed to the center of the MBP and steeply decaying measurements are found at increasing distances from the center.

Investigating the intensity excess of MBPs, which correspond to all MBPs containing strong $\left(>3 \mathrm{~km} \mathrm{~s}^{-1}\right)$ downflows and $G$ band inter-granular brightenings, reveals a correlation with the underlying magnetic field concentrations. Figure 4 reveals how the intensity excess of $G$-band, Ca II $\mathrm{K}$, and $\mathrm{Na}_{\text {I }} \mathrm{D}_{1}$ observations varies as a function of the magnetic flux density, $\Phi$, defined as the total magnetic field strength divided by the number of occupied pixels. The excess intensity, $\Delta I$, is determined as an averaged pixel intensity for the entire MBP, following a subtraction of intensities equal to the modal value observed in quiet areas (central panels of Figure 2). For $G$-band images, MBPs are isolated from granules and inter-granular lanes using the automated routines of Crockett et al. (2009). These isolated pixels also provide the structures used to determine the magnetic flux density from the line-of-sight magnetograms. Na I $D_{1}$ core MBP observations consist of a bright central structure, surrounded by a dimmer halo, resulting from radiative scattering (Leenaarts et al. 2010). The central MBP is isolated from the halo by utilizing simultaneous $\mathrm{Na}_{\mathrm{I}} \mathrm{D}_{1}$ core velocity maps. The lower-right panel of Figure 2 reveals how the downdraft velocities drop to approximately zero at the edge of the bright central MBP. Therefore, pixels where the downdraft velocity is non-zero are used to isolate the central MBP regions, thus minimizing contributions to the excess intensity caused by radiative scattering, which is only weakly dependent on the magnetic field strength.

In the case of $G$-band observations (upper panel of Figure 4), a linear dependence observed in the $\log -\log$ plot is in agreement with Beck et al. (2007), who detect identical trends for $G$ band brightenings in active region moats. Through analyses of network bright points ( $>3^{\prime \prime}$ in diameter) and large-scale active region structures ( $>15^{\prime \prime}$ in diameter), Cauzzi et al. (2000) and Schrijver et al. (1989a) were able to demonstrate a similar trend for these features when observed in the $\mathrm{Na}_{\text {I }} \mathrm{D}_{2}$ and $\mathrm{Ca}$ II $\mathrm{K}$ lines, respectively. Indeed, for the magnetic flux densities presented here (above $\approx 150 \mathrm{G}$ in the case of sodium), the calcium, $G$-band and sodium excesses (Figure 4 ) are best fitted by a power law of the type $\Delta I \propto \Phi^{\beta}$, where $\beta$ is the powerlaw exponent. Here, we determine $\beta=0.61 \pm 0.02$ for $\mathrm{Ca}$ II $\mathrm{K}$ observations and $\beta=0.59 \pm 0.03$ in the case of $\mathrm{Na}$ I $\mathrm{D}_{1}$ data. These values of power-law exponent are in agreement with the previous $\mathrm{NaI}_{\mathrm{I}} \mathrm{D}_{2}$ study of Cauzzi et al. (2000) and remain consistent with exponents found in $\mathrm{Ca}$ II $\mathrm{K}$ investigations (Skumanich et al. 1975; Nindos \& Zirin 1998). While these consistencies with previously published work may seem initially superfluous, the spatial scale over which these results agree is extraordinary. Schrijver et al. (1989a) investigated large-scale magnetic structures contained within an active region, often with spatial sizes approaching $70^{\prime \prime}$ in diameter. The MBPs investigated here consist of structures as small as $\approx 0 ! 2$ in diameter, more than 2 orders of magnitude smaller than those studied previously.

At magnetic flux densities below $\approx 150 \mathrm{G}$, there is an appreciable "turnoff" from the power-law fit applied to the Na I $\mathrm{D}_{1}$ observations. The lower panel of Figure 4 reveals how the $\mathrm{Na}$ I 

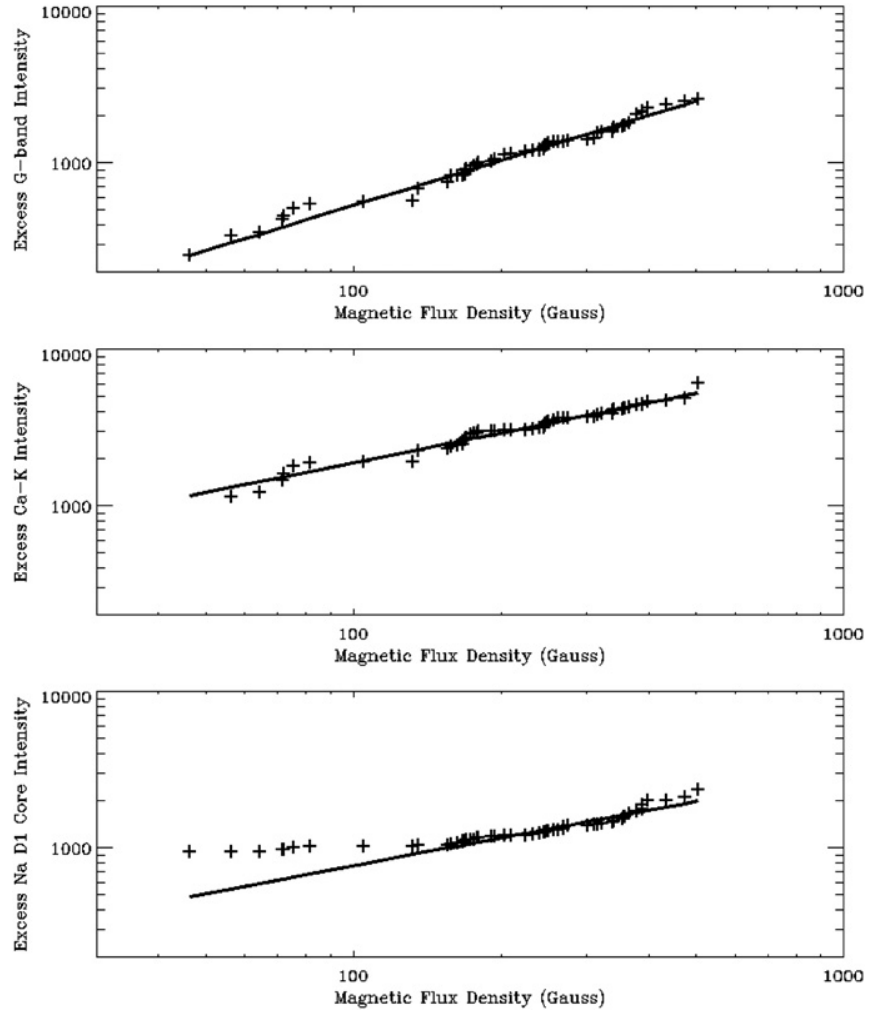

Figure 4. Intensity excess for each MBP containing downdraft velocities exceeding $3 \mathrm{~km} \mathrm{~s}^{-1}$, plotted as a function of MBP-averaged magnetic flux density for $G$-band (top), Ca II K (middle), and Na I $\mathrm{D}_{1}$ (bottom) observations, and displayed on logarithmic axes. In each panel, power-law fits have been applied to the data which best minimizes the chi-squared error statistic.

$\mathrm{D}_{1}$ intensity remains elevated, even as the magnetic flux density begins to diminish. Through analysis of Ca II $\mathrm{K}$ observations, Schrijver et al. (1989a) suggest that large deviations in linecore intensity, at relatively small magnetic flux densities, may be a result of a large inclination of the magnetic flux tubes, or small-scale energetic phenomena. Inclination effects on the data presented here are not believed to be significant for two reasons. First, the inclination angle of MBPs, with respect to the vertical, is typically less than $10^{\circ}$ (Sánchez Almeida \& Martínez Pillet 1994). Second, due to the observations being acquired at the disk center, line-of-sight effects are minimized. This allows photospheric structures to remain cospatial when observed in chromospheric passbands, and since no feature migration is found between $G$-band and $\mathrm{Ca}$ II $\mathrm{K}$ atmospheric heights, further solidifies the presence of minimal inclination angles. Additionally, the intensities of MBPs corresponding to $G$-band, Ca II K, and $\mathrm{Na}$ I $\mathrm{D}_{1}$ observations show no temporal variations that may be suggestive of microflare (Jess et al. 2010a) and/or Ellerman bomb (Ellerman 1917) activity. Through examination of photospheric $G$-band MBPs, we observe the physical area of such structures to reduce as the magnetic field becomes weaker, corroborating the observational results of Ishikawa et al. (2007). Contrarily, we find MBPs, which correspond to magnetic flux densities below the turnoff at $\approx 150 \mathrm{G}$, show similar two-dimensional areas when viewed in the $\mathrm{Na}$ I $\mathrm{D}_{1}$ line core. An average Na I $\mathrm{D}_{1}$ core area of $0.11 \pm 0.01 \mathrm{Mm}^{2}(47 \pm$ 4 pixels) is found for these structures, suggesting that their excess intensity plateau below $\approx 150 \mathrm{G}$ may be related to the physical expansion of magnetic flux tubes. Solanki et al. (1999) utilize models based upon infrared spectral lines to infer how small magnetic flux tubes expand more rapidly as a function of atmospheric height, when compared with larger magnetic structures. Thus, in order to maintain relatively constant physical sizes present in $\mathrm{Na}$ I $\mathrm{D}_{1}$ observations, it is necessary for the physically smaller (and weaker) magnetic field concentrations in the photosphere to expand at a greater rate. To quantify this result, we find that MBPs at the lowest magnetic flux densities $(\approx 50 \mathrm{G})$ show a $\sim 76 \%$ increase in area between $G$-band and $\mathrm{Na}$ I $\mathrm{D}_{1}$ observations, compared to a $\sim 44 \%$ increase for MBPs near the $\approx 150 \mathrm{G}$ turnoff.

A discontinuity in the correlation between the magnetic field and $\mathrm{Na}$ I $\mathrm{D}_{1}$ intensity, for magnetic flux densities below $\approx 150 \mathrm{G}$, suggests that these MBP intensities are independent of the underlying magnetic field strength. Combining $\mathrm{Na}$ I $\mathrm{D}_{1}$ observations with three-dimensional non-LTE simulations, Leenaarts et al. (2010) conclude that the Na I $\mathrm{D}_{1}$ line is not chromospheric in nature and instead samples the atmospheric layer below the magnetic canopy, where magnetoacoustic shocks are believed to dominate. Our findings may therefore indicate a two-component heating process: one which is primarily acoustic and dominates the heating of low magnetic field strength areas, while the other process is predominantly magnetic in nature. Within the presented data, MBPs with velocities exceeding $3 \mathrm{~km} \mathrm{~s}^{-1}$ represent the dominant population. However, as MBPs can exist with velocities below this threshold, their excess intensity behavior, as a function of the magnetic flux density, may differ as a result. We note that two-component heating models have previously been proposed to interpret observed chromospheric flux densities in the most inactive solar-type stars (Schrijver et al. 1989b; Mathioudakis \& Doyle 1992).

\section{CONCLUDING REMARKS}

Joint observations, acquired with the Rapid Oscillations in the Solar Atmosphere (ROSA) and Interferometric BIdimensional Spectrometer (IBIS) imaging systems, have enabled unprecedented views of solar MBPs. These data, obtained simultaneously with high spatial, temporal, and spectral resolutions, have revealed how MBPs manifesting in the Na I $D_{1}$ core are found to preferentially exist in regions containing downflows, in addition to cospatial underlying magnetic field concentrations. Downdrafts found within Na I $\mathrm{D}_{1}$ bright points exhibit speeds of up to $7 \mathrm{~km} \mathrm{~s}^{-1}$, with preferred structural symmetry in intensity, magnetic field, and velocity profiles about the center of the MBP. Excess intensities associated with $G$-band and Ca II K observations of MBPs demonstrating downdrafts exceeding $3 \mathrm{~km} \mathrm{~s}^{-1}$ reveal a power-law trend when plotted as a function of the magnetic flux density. However, Na I $\mathrm{D}_{1}$ observations of the same magnetic features indicate an intensity plateau at weak magnetic field strengths below $\approx 150 \mathrm{G}$. We interpret this phenomena as the physical expansion of magnetic flux tubes, with weak field strengths ( $\approx 50 \mathrm{G}$ ) expanding by $\sim 76 \%$, compared to $\sim 44 \%$ expansion for structures at higher magnetic field strengths $(\approx 150 \mathrm{G})$.

D.B.J. thanks STFC for a Post-Doctoral Fellowship. D.J.C. thanks CSUN for start-up funding. P.J.C. is grateful to NIDEL for a $\mathrm{PhD}$ studentship. F.P.K. thanks AWE Aldermaston for the William Penney Fellowship.

\section{REFERENCES}

Aimanova, G. K., \& Gulyaev, R. A. 1976, SvA, 20, 201

Athay, R. G., \& Canfield, R. C. 1969, ApJ, 156, 695

Beck, C., Bellot Rubio, L. R., Schlichenmaier, R., \& Sütterlin, P. 2007, A\&A, 472, 607 
Beebe, H. A., \& Johnson, H. R. 1969, Sol. Phys., 10, 79

Carlsson, M. 1986, Uppsala Astronomical Observatory Reports, 33

Cauzzi, G., Falchi, A., \& Falciani, R. 2000, A\&A, 357, 1093

Cauzzi, G., et al. 2008, A\&A, 480, 515

Cavallini, F. 2006, Sol. Phys., 236, 415

Crockett, P. J., Jess, D. B., Mathioudakis, M., \& Keenan, F. P. 2009, MNRAS, 397,1852

Edmonds, F. N., Jr., \& Hsu, J.-C. 1983, Sol. Phys., 83, 217

Eibe, M. T., Mein, P., Roudier, T., \& Faurobert, M. 2001, A\&A, 371, 1128

Ellerman, F. 1917, ApJ, 46, 298

Finsterle, W., Jefferies, S. M., Cacciani, A., Rapex, P., \& McIntosh, S. W. 2004, ApJ, 613, L185

Ishikawa, R., et al. 2007, A\&A, 472, 911

Jess, D. B., Mathioudakis, M., Browning, P. K., Crockett, P. J., \& Keenan, F. P. 2010a, ApJ, 712, L111

Jess, D. B., Mathioudakis, M., Christian, D. J., Keenan, F. P., Ryans, R. S. I., \& Crockett, P. J. 2010b, Sol. Phys., 261, 363

Jess, D. B., Mathioudakis, M., Crockett, P. J., \& Keenan, F. P. 2008, ApJ, 688, L119

Jess, D. B., McAteer, R. T. J., Mathioudakis, M., Keenan, F. P., Andic, A., \& Bloomfield, D. S. 2007, A\&A, 476, 971
Langangen, Ø., Rouppe van der Voort, L., \& Lin, Y. 2008, ApJ, 673, 1201

Leenaarts, J., Rutten, R. J., Reardon, K., Carlsson, M., \& Hansteen, V. 2010, ApJ, 709,1362

Mathioudakis, M., \& Doyle, J. G. 1992, A\&A, 262, 523

Nindos, A., \& Zirin, H. 1998, Sol. Phys., 179, 253

Reardon, K. P., \& Cavallini, F. 2008, A\&A, 481, 897

Rimmele, T. R. 2004, Proc. SPIE, 5490, 34

Roudier, T., Malherbe, J. M., Moity, J., Rondi, S., Mein, P., \& Coutard, C. 2006, A\&A, 455, 1091

Sánchez Almeida, J., \& Martínez Pillet, V. 1994, ApJ, 424, 1014

Schrijver, C. J., Cote, J., Zwaan, C., \& Saar, S. H. 1989a, ApJ, 337, 964

Schrijver, C. J., Dobson, A. K., \& Radick, R. R. 1989b, ApJ, 341, 1035

Simoniello, R., Jiménez-Reyes, S. J., García, R. A., \& Pallé, P. L. 2008, Astron. Nachr., 329, 494

Skumanich, A., Smythe, C., \& Frazier, E. N. 1975, ApJ, 200, 747

Slaughter, C. D., \& Wilson, A. M. 1972, Sol. Phys., 24, 43

Solanki, S. K., Finsterle, W., Rüedi, I., \& Livingston, W. 1999, A\&A, 347, L27

Tomita, Y. 1960, PASJ, 12, 524

Vernazza, J. E., Avrett, E. H., \& Loeser, R. 1981, ApJS, 45, 635

Weigelt, G., \& Wirnitzer, B. 1983, Opt. Lett., 8, 389

Wöger, F., von der Lühe, O., \& Reardon, K. 2008, A\&A, 488, 375 